\title{
Phantom Shocks Associated With a Wearable Cardioverter Defibrillator
}

\author{
Paul Delle Donna ${ }^{a}$, Luka Petrovic ${ }^{a}$, Umair Nasir ${ }^{\mathrm{a}}$, Ahmed Ahmed ${ }^{\mathrm{a}}$, \\ Giselle Alexandra Suero-Abreu ${ }^{a, b}$
}

\begin{abstract}
Wearable cardioverter defibrillators (WCDs) are external devices capable of continuous cardiac rhythm monitoring as well as automatic detection and defibrillation of potentially life-threatening arrhythmias such as ventricular tachycardia (VT) and ventricular fibrillation (VF). They are an alternative approach for patients when an implantable cardioverter defibrillator (ICD) is not appropriate. Although treatment with ICD is considered highly effective for the primary and secondary prevention of sudden cardiac death (SCD) in high-risk patients susceptible to VT and VF, patients may still experience psychological difficulties such as fear of shock, avoidance of normal behaviors and reduced quality of life. One of these phenomena is phantom shock (PS), which is defined as a perception of having received a shock with no evidence of recorded defibrillation upon device interrogation. While PS has been reported in the ICD literature, to the best of our knowledge, we present the first known case of WCD-related PS. We also present a review of the current literature to explore the prevalence of PS, the factors associated with its pathogenesis and interventional studies aimed at reducing its occurrence. We highlight this case because PS is considered a phenomenon that few recognize, which should be discriminated from real device shocks before clinicians initiate treatment, device reprogramming or device discontinuation. We describe the psychosocial factors associated with PS to emphasize the importance of managing any associated psychiatric disorders and psychosocial factors both before and after initiation of device treatment.
\end{abstract}

Keywords: Phantom shock; Wearable cardioverter defibrillator; Implantable cardioverter defibrillator; Sudden cardiac death

\section{Introduction}

One of the most feared complications of many cardiac diseases

Manuscript submitted October 9, 2020, accepted November 7, 2020

Published online December 30, 2020

aDepartment of Medicine, Rutgers New Jersey Medical School, Newark, NJ, USA

${ }^{b}$ Corresponding Author: Giselle Alexandra Suero-Abreu, Department of Medicine, Rutgers New Jersey Medical School, 150 Bergen Street, Newark, NJ 07107, USA. Email: giselle.suero@rutgers.edu

doi: https://doi.org/10.14740/jmc3606 is sudden cardiac death (SCD). Prompt cardiac defibrillation has been shown as an effective means of terminating ventricular tachycardia (VT) and ventricular fibrillation (VF), thus preventing SCD when these arrhythmias occur. Cardioverter defibrillators are considered the best available option for the primary and secondary prevention of SCD in patients with cardiac conditions that place them at a higher risk for SCD, such as hypertrophic cardiomyopathy, heart failure with reduced ejection fraction (HFrEF), Brugada syndrome, arrhythmogenic right ventricular dysplasia (ARVD), cardiac sarcoidosis and long QT syndrome (Table 1) [1-4].

ICDs are the most widely used and studied devices for SCD prevention, with the first device implanted in 1980 by Mirowski et al [2]. As shown in the MADIT II trial, prophylactic ICD placement is highly effective in reducing all-cause mortality by $31 \%$ in post-myocardial infarction (MI) patients with an ejection fraction less than or equal to $30 \%$ [3]. However, a subset of high-risk patients who meet criteria for ICD placement are unable to undergo immediate or even future ICD implantation for multiple reasons. Some contraindications for immediate ICD placement include MI within the preceding 3 months and non-adherence directed medical treatment (GDMT) in heart failure patients for 3 months prior to ICD implantation. Additionally, bacteremia, fungemia, prior ICD infection and patients who are poor surgical candidates preclude immediate ICD placement [3,4]. As a result, these patients have been left to rely on emergency medical services in the event that they develop a ventricular tachyarrhythmia. In response, wearable cardioverter defibrillators (WCDs) were developed and FDA approved in 2001 as a bridge to permanent ICD placement or cardiac transplant. Since then, WCDs have also been approved for additional indications such as an alternative for SCD prevention in patients who are unable or unwilling to undergo an invasive procedure for ICD placement (Table 2) $[5,6]$.

A WCD is a wearable external device that serves the same role as an ICD. The device is composed of a vest that the patient wears externally. On its inner surface, there are electrodes that sense the electrical activity of the heart and deliver this information to the processor which analyzes the continuous electrocardiogram (EKG). If VT or VF is detected, thus necessitating a therapeutic shock, the WCD will first alert the patient with vibratory and auditory warnings that a shockable rhythm has been detected. The patient can abort the shock by pressing two buttons on the unit simultaneously. If the patient does not press the buttons, a shock will be delivered via electrodes on 
Table 1. Indications for ICD Placement for Primary and Secondary Prevention of SCD [4]

\begin{abstract}
Primary prevention of SCD
MI within the past 40 days and $\mathrm{LVEF} \leq 35 \%$

Patients with NICM with LVEF $\leq 35 \%$ after 3 months of optimized GDMT

HF patients who are candidates for CRT with BVICDa

Patients with congenital long QT syndrome with recurrent symptoms despite medical therapy

High-risk patients with hypertrophic cardiomyopathy

High-risk patients with Brugada syndrome, polymorphic VT and other channelopathies

Secondary prevention of SCD

Prior episode of survived VF/VT or sustained hemodynamically unstable VT without an identifiable reversible cause

Patients with episodes of spontaneously sustained VT in the presence of heart disease

aLVEF $\leq 35 \%$, on maximal GDMT for at least 3 months and has intraventricular conduction delay of more than $120 \mathrm{~ms}$. ICD: implantable cardioverter defibrillator; SCD: sudden cardiac death; MI: myocardial infarction; LVEF: left ventricular ejection fraction; NICM: non-ischemic cardiomyopathy; GDMT: goal-directed medical therapy; CRT: cardiac resynchronization therapy; BVICD: biventricular implantable cardioverter defibrillator; VT: ventricular tachycardia; VF: ventricular fibrillation.
\end{abstract}

the inside of the vest [7].

As with all medical devices, WCDs are not without the risk of adverse events. One known complication in patients using ICD and WCD is inappropriate shocks (ISs), which are device-delivered electrical shocks for a reason other than actual VT or VF. ISs are most commonly the result of device misinterpretation of the patient's cardiac rhythm and can be painful and traumatizing to the patient. Another adverse event described in the literature is phantom shock (PS). PS is a patient-reported episode during which the patient claims to have experienced a shock delivered by their ICD unit, yet upon device interrogation, no evidence of a shock is present [7-10]. Due to the perception of an actual shock, it presents an understandable concern for the patient and often prompts them to seek emergent clinical evaluation. To date, the occurrence, etiology and treatment of PS have only been investigated in ICD populations. Although a PS has never been reported in the literature by a patient wearing a WCD, it is reasonable to suspect that PS could also occur with a WCD given its similar function and delivered therapy and here we describe the first known case of PS in a patient wearing a WCD.

\section{Case Report}

A 54-year-old man with a past medical history of hypertension, HFrEF (left ventricular ejection fraction (LVEF) 25\%), MI 3 weeks prior, generalized anxiety disorder (GAD), major depressive disorder (MDD), polysubstance abuse (alcohol, cocaine and tobacco with recent cocaine and alcohol use prior to presentation) and chronic back pain presented to the emergency department (ED) complaining that his WCD had discharged. Three weeks earlier, he had been diagnosed with new onset HFrEF secondary to coronary artery disease. At that time, a transthoracic echocardiogram showed inferior wall motion abnormalities and a left heart catheterization revealed a $75 \%$ narrowing of the right coronary artery. Balloon angioplasty of the artery was performed and a drug eluding stent was placed. The patient was started on GDMT for heart failure and received a WCD because he did not qualify for ICD placement due to his recent MI (within the past 40 days).

Table 2. Indications for WCD Placement $[5,6]$

Patient populations

Patients with NICM and LVEF $\leq 35 \%$, NYHA class II-III less than 3 months of GDMT

Patients with ICM and LVEF $\leq 35 \%$, NYHA class II-III who cannot undergo ICD placement, within 40 days of MI or 90 days of a CABG procedure

Patients awaiting ICD reimplantation after device infection or dislodgement

Patients who are not good candidates for ICD or are not good surgical candidates

Patients who prefer less invasive means for SCD prophylaxis

Patients with a possibly reversible cause of reduced LVEF (MC, PPCM, SIC)

Patients with terminal HFrEF awaiting to undergo cardiac transplant in near future

WCD: wearable cardioverter defibrillator; NICM: non-ischemic cardiomyopathy; LVEF: left ventricular ejection fraction; NYHA: New York Heart Association; GDMT: goal-directed medical therapy; ICM: ischemic cardiomyopathy; ICD: implantable cardioverter defibrillator; MI: myocardial infarction; CABG: coronary artery bypass graft surgery; SCD: sudden cardiac death; MC: myocarditis; PPCM: peripartum cardiomyopathy; SIC: stress-induced cardiomyopathy; HFrEF: heart failure with reduced ejection fraction. 
According to the patient, while socializing with friends outside his home, he felt a sudden jolting sensation for a total of five times which was witnessed by the bystanders. This was followed by the acute onset of dyspnea, diaphoresis, lightheadedness and palpitations. Emergency medical service (EMS) was called and he was transported to the hospital for further evaluation. On arrival to the ED, the patient was still wearing the WCD and reported the episode of shock from the device. Vitals were within normal limits with the exception of a heart rate of 107. Laboratory workup showed complete blood count, complete metabolic count and electrolytes within normal limits. Pro-brain natriuretic peptide (pro-BNP) was elevated at 5,000 while troponins, creatine kinase and creatine kinase-MB were all within normal limits. An EKG showed sinus tachycardia, left atrial enlargement and Q waves in an infero-lateral distribution due to prior MI that were unchanged from prior EKGs. Chest X-ray showed mild pulmonary congestion. The patient was treated with furosemide $40 \mathrm{mg} \mathrm{IV}$, aspirin $325 \mathrm{mg}$ PO, rosuvastatin $20 \mathrm{mg}$ PO and carvedilol $25 \mathrm{mg}$ PO. Upon evaluation by the cardiology service, the patient was anxious but in no acute distress, and a physical exam revealed trace bilateral lower extremity pitting edema and an $\mathrm{S} 3$ heart sound. The patient was still wearing his WCD. He was admitted to the telemetry service for diuresis, further monitoring and interrogation of the WCD which revealed no recorded shocks. As a result, PS from the WCD was added to the differential diagnosis. The patient remained stable and the rest of the workup and telemetry monitoring was unremarkable. Upon further questioning, the patient admitted that he had been "nervous about the device" since he began wearing it 3 months prior to presentation and that he "may have overreacted" to the device beeping. The patient was discharged on hospital day 2 with instructions to continue his GDMT for HFrEF and wearing his $\mathrm{WCD}$ as it was functioning normally. A follow-up appointment with the cardiology service was set to have a repeat transthoracic echocardiogram performed in 3 months. The patient was advised to seek psychological evaluation and to initiate alcohol and illicit substance cessation through an inpatient or outpatient substance abuse rehabilitation program, but the patient declined.

\section{Discussion}

WCDs have become an important option for SCD prevention in patients who are unable to receive an ICD and their effectiveness has been studied in several clinical trials. In a study conducted by Epstein et al, the 12-month cumulative survival rate for WCD patients at increased risk of SCD postMI was 93\% [11]. The success rate of therapeutically appropriate WCD shocks has also been studied by multiple groups. A meta-analysis of all WCD trials conducted by Agarwal et al reported that appropriate shocks delivered by WCDs had a success rate at terminating ventricular arrhythmias ranging from $75 \%$ to $80 \%$ [12]. Chung et al reported a trial of 3,569 patients directly compared WCDs to ICDs and showed WCDs to be an acceptable alternative for SCD prevention in patients with need of an ICD but contraindications for its placement, showing no statistical difference in mortality between the ICD and WCD groups at any time interval after device implementation [3].

PS in an ICD patient was first reported in 1992 and has been studied in the ICD population; however, to the best of our knowledge, PS has never been reported in the WCD literature. The incidence of PS in ICD patients ranges from 5.1\% to $25.33 \%$, but the incidence of PS in the WCD population is unknown [8-10]. Although there is a prior lack of evidence of PS in WCD population, it is plausible that WCD patients could experience PS given the similarities between ICD and WCD use, and this case supports that hypothesis. However, further studies based on data from the WCD patient population are needed to determine the incidence of PS in WCD patients. ICD studies investigating PS have attempted to examine the predisposing factors, etiology and possible treatment for PS. Depression, anxiety, post-traumatic stress disorder (PTSD), prior device shocks, illicit drug use and time since ICD placement have all been hypothesized to contribute to PS. Identifying and treating these underlying conditions before device implementation may reduce patient's morbidity, the number of ED visits and hospital admissions. More importantly, it would increase quality of life following device placement and improve adherence to WCD use [13-15].

The relationship between predisposing factors and the occurrence of PS lacks consistency across the published studies. In a study by Berg et al, there was no statistically significant relationship between PS, age, gender, mental health and number of actual delivered shocks, both appropriate and inappropriate [8]. However, a larger study including 629 patients conducted by Kraaier et al showed that patients with an ICD implanted for primary prevention of SCD who experienced PS had a higher incidence of underlying atrial fibrillation and New York Heart Association (NYHA) class II heart failure [10]. The patient in our case had class II NYHA HFrEF as an associated medical comorbidity for PS. Furthermore, mental health comorbidities have been a focus of PS studies in the ICD literature. It has been hypothesized that ICD patients who experienced PS have higher levels of anxiety, depression and PTSD that may somatically manifest as a patient-perceived shock. However, the literature is inconsistent with regard to the relationship between PS and mental illness. Berg et al showed no relationship between the occurrence of PS and mental illness [8]. Further, Pedersen et al have shown that patients maintained their pre-implantation level of psychiatric functionality, yet recognized that a small subset of the ICD population was at risk for poor adaptation after device implantation related to a history of prior ICD shocks, atrial fibrillation and known left ventricular dysfunction [16-18]. Jacob et al showed that 31\% of the patients who experienced PS also had clinical depression, $10 \%$ had a family history of psychiatric disorders, $18.4 \%$ were taking antidepressants and $42.1 \%$ had history of cocaine use [13]. Our patient exhibited many of the predisposing conditions which have been associated with PS. He had a prior diagnosis of untreated GAD and MDD. Concomitantly he had a history of substance abuse disorder and was actively consuming cocaine and abusing alcohol that may have contributed to his psychiatric illnesses. In addition, cocaine use is known to cause coronary vasospasm and chest pain which can be mis- 
taken for a shock.

One possibility is that the anxiety PS patients experience is an exacerbation of their underlying, preexisting, organic psychiatric conditions which predispose them to experience PS after device implementation. In addition, device implantation itself has been thought to directly precipitate depression and anxiety, leading to post-implantation mental illness. After the device is prescribed, patients are more aware of the severity of their condition and worry that there is a high probability that they will experience an episode of SCD. Depression and anxiety may have also been attributable to a limited patient understanding about the device itself and how to make appropriate post-implantation life modifications. This highlights the importance of patient counseling regarding these devices pre- and post-implantation and ongoing communication between the physician and patient. Varghese et al showed that $22 \%$ of patients felt that they did not receive adequate information before having their ICD placed and $26 \%$ of ICD recipients would prefer ongoing psychosocial support, further suggesting the importance of these practices [14]. Humphrey et al suggested multiple types of effective psycho-social interventions such as patient education, mindfulness training, the development of anxiety-relieving coping mechanisms and goal setting as possible interventions in the prevention of PS in the ICD population [15]. Screening for depression, anxiety and PTSD may also be of value before device implantation, since when underlying illnesses are identified, prompt initiation of proper medical and psychological therapy can decrease the contribution of these disorders to the development of PS. Similarly, screening and treatment for any substance abuse disorders may also be useful in the prevention of PS in patients who are being considered for ICD or WCD placement.

Although there is a lack of large interventional studies, multiple cases of successful medical treatment of PS have been reported in the ICD literature. Hairston et al reported that a combination of antidepressants and cognitive behavioral therapy was an effective treatment for PS in a patient with concurrent adjustment disorder with mixed anxiety and depressed mood in addition to cocaine use disorder [19]. A second case of nocturnal PS in an ICD patient was successfully treated with zolpidem [20]. Hamner et al described successful treatment of two patients with PS and PTSD with antidepressants and psychotherapy [21]. Lastly, beta-blockers have also been shown to help with symptoms of PTSD in patients that experienced prior shocks [22].

In conclusion, it is clear that treating patients with ICDs and WCDs for primary or secondary prevention is very complex and goes beyond device implantation. A multidisciplinary approach should be implemented when an ICD or WCD is being considered in order to address underlying comorbidities and initiate appropriate treatment and patient education. It is unclear whether there are effective ways to reduce or prevent PS. However, screening and treatment for underlying comorbidities that would predispose to PS has yet to become part of the device delivery process. Appropriate treatment with medication, psychotherapy and substance abuse programs pre- and post-device implantation may prove successful in the prevention of PS and may improve adherence WCD use in patients. In future, large randomized controlled studies are needed to prove the effectiveness of these therapeutic interventions.

\section{Conclusions}

PS is a common phenomenon in the ICD patient population and must be considered when a patient presents in an acute setting for a perceived device shock. This case report shows that PS also occurs in the WCD patient population. Awareness of PS as a possible diagnosis in WCD recipients will allow physicians to consider PS as a differential diagnosis in the setting of patient-reported shock. In such cases, patients can be reassured that their device is functioning properly, preventing potential hospital admissions. A multidisciplinary approach including cardiology, psychology and addiction medicine should be considered when evaluating a patient in need of a WCD or ICD to improve quality of life and address potential incidents of PS.

\section{Acknowledgments}

None to declare.

\section{Financial Disclosure}

None to declare.

\section{Conflict of Interest}

None to declare.

\section{Informed Consent}

The manuscript has been sufficiently de-identified to protect the identity of each patient. Patients have since died and unable to provide informed consent.

\section{Author Contributions}

Each individual named as an author meets the journal's criteria for authorship. Authors edited the manuscript, contributed with review of the literature and discussion section; PDD contributed to patient care and conceptualization, writing of manuscript, design of tables and editing; LP contributed to writing and editing of the manuscript, and discussion section; UM contributed to review of the literature and discussion section; AA contributed to review of literature and editing of the manuscript; GASA contributed to conceptualization, review of literature, manuscript editing and writing.

\section{Data Availability}

The authors declare that data supporting the findings of this study are available within the article. 


\section{References}

1. Nichol G, Sayre MR, Guerra F, Poole J. Defibrillation for ventricular fibrillation: a shocking update. J Am Coll Cardiol. 2017;70(12):1496-1509.

2. Mirowski M, Reid PR, Mower MM, Watkins L, Gott VL, Schauble JF, Langer A, et al. Termination of malignant ventricular arrhythmias with an implanted automatic defibrillator in human beings. $\mathrm{N}$ Engl J Med. 1980;303(6):322-324.

3. Moss AJ, Zareba W, Hall WJ, Klein H, Wilber DJ, Cannom DS, Daubert JP, et al. Prophylactic implantation of a defibrillator in patients with myocardial infarction and reduced ejection fraction. N Engl J Med. 2002;346(12):877-883.

4. Al-Khatib SM, Stevenson WG, Ackerman MJ, Bryant WJ, Callans DJ, Curtis AB, Deal BJ, et al. 2017 AHA/ ACC/HRS guideline for management of patients with ventricular arrhythmias and the prevention of sudden cardiac death: Executive summary: A Report of the American College of Cardiology/American Heart Association Task Force on Clinical Practice Guidelines and the Heart Rhythm Society. Heart Rhythm. 2018;15(10):e190-e252.

5. Barraud J, Cautela J, Orabona M, Pinto J, Missenard O, Laine M, Thuny F, et al. Wearable cardioverter defibrillator: Bridge or alternative to implantation? World J Cardiol. 2017;9(6):531-538.

6. Lamichhane M, Safadi A, Surapaneni P, Salehi N, Thakur RK. Use of the wearable cardioverter defibrillator in high-risk populations. Curr Cardiol Rep. 2016;18(8):78.

7. Francis J, Reek S. Wearable cardioverter defibrillator: a life vest till the life boat (ICD) arrives. Indian Heart J. 2014;66(1):68-72.

8. Berg SK, Moons P, Zwisler AD, Winkel P, Pedersen BD, Pedersen PU, Svendsen JH. Phantom shocks in patients with implantable cardioverter defibrillator: results from a randomized rehabilitation trial (COPE-ICD). Europace. 2013;15(10):1463-1467.

9. Prudente LA. Phantom shock in a patient with an implantable cardioverter defibrillator: case report. Am J Crit Care. 2003;12(2):144-146.

10. Kraaier K, Starrenburg AH, Verheggen RM, van der Palen J, Scholten MF. Incidence and predictors of phantom shocks in implantable cardioverter defibrillator recipients. Neth Heart J. 2013;21(4):191-195.

11. Epstein AE, Abraham WT, Bianco NR, Kern KB, Mirro $\mathrm{M}$, Rao $\mathrm{SV}$, Rhee $\mathrm{EK}$, et al. Wearable cardioverter- defibrillator use in patients perceived to be at high risk early post-myocardial infarction. J Am Coll Cardiol. 2013;62(21):2000-2007.

12. Agarwal M, Narcisse D, Khouzam N, Khouzam RN. Wearable Cardioverter Defibrillator "The Lifevest": Device Design, Limitations, and Areas of Improvement. Curr Probl Cardiol. 2018;43(2):45-55.

13. Jacob S, Panaich SS, Zalawadiya SK, McKelvey G, Abraham G, Aravindhakshan R, Sears SF, et al. Phantom shocks unmasked: clinical data and proposed mechanism of memory reactivation of past traumatic shocks in patients with implantable cardioverter defibrillators. J Interv Card Electrophysiol. 2012;34(2):205-213.

14. Varghese S, Geller JC, Ohlow MA. Phantom shocks in implantable cardioverter-defibrillator recipients: impact of education level, anxiety, and depression. Herzschrittmacherther Elektrophysiol. 2019;30(3):306-312.

15. Humphreys NK, Lowe R, Rance J, Bennett PD. Living with an implantable cardioverter defibrillator: The patients' experience. Heart Lung. 2016;45(1):34-40.

16. Hoogwegt MT, Kupper N, Jordaens L, Pedersen SS, Theuns DA. Comorbidity burden is associated with poor psychological well-being and physical health status in patients with an implantable cardioverter-defibrillator. Europace. 2013;15(10):1468-1474.

17. Pedersen SS, Hoogwegt MT, Jordaens L, Theuns DA. Procedure- and device-related complications and psychological morbidity in implantable cardioverter defibrillator patients. Int J Cardiol. 2013;168(1):606-607.

18. Hoogwegt MT, Widdershoven JW, Theuns DA, Pedersen $\mathrm{SS}$. Information provision, satisfaction and emotional distress in patients with an implantable cardioverter-defibrillator. Int J Cardiol. 2014;177(2):586-588.

19. Hairston DR, de Similien RH, Himelhoch S, Forrester A. Treatment of phantom shocks: A case report. Int J Psychiatry Med. 2019;54(3):181-187.

20. House LM, McKay RE, Eagan JT, Jr., McCormick ZL. Nocturnal phantom shock cessation with zolpidem. Heart Lung. 2018;47(1):76-79.

21. Hamner M, Hunt N, Gee J, Garrell R, Monroe R. PTSD and automatic implantable cardioverter defibrillators. Psychosomatics. 1999;40(1):82-85.

22. Bhuvaneswar CG, Ruskin JN, Katzman AR, Wood N, Pitman RK. Pilot study of the effect of lipophilic vs. hydrophilic beta-adrenergic blockers being taken at time of intracardiac defibrillator discharge on subsequent PTSD symptoms. Neurobiol Learn Mem. 2014;112:248-252. 